\title{
Practical design issues for snapshot Mueller matrix spectropolarimetry
}

Hagen, Nathan, Chrysler, Benjamin, Otani, Yukitoshi

Nathan Hagen, Benjamin D. Chrysler, Yukitoshi Otani, "Practical design issues for snapshot Mueller matrix spectropolarimetry," Proc. SPIE 11132, Polarization Science and Remote Sensing IX, 111320H (6 September 2019); doi: $10.1117 / 12.2525006$

Event: SPIE Optical Engineering + Applications, 2019, San Diego, California, United States 


\title{
Practical design issues for snapshot Mueller matrix spectropolarimetry
}

\author{
Nathan Hagen ${ }^{a}$, Benjamin D. Chrysler ${ }^{b}$, and Yukitoshi Otani ${ }^{a}$ \\ ${ }^{a}$ Utsunomiya University, Department of Optical Engineering, Center for Optical Research and \\ Engineering (CORE), Utsunomiya, Tochigi 321-8585 Japan \\ ${ }^{b}$ University of Arizona, College of Optical Sciences, 1630 E. University Blvd., Tucson, Arizona \\ 85721, USA
}

\begin{abstract}
We discuss practical design constraints for snapshot Mueller matrix spectropolarimeters, and reveal a robustness problem with existing designs. By carefully choosing the ratios of thicknesses between the four thick retarders used in these systems, we can avoid requiring extremely tight tolerances, though at a cost in overall bandwidth. We provide example designs and quantify the robustness-resolution tradeoff.
\end{abstract}

Keywords: Polarization, spectropolarimetry, Mueller matrix

\section{INTRODUCTION - CHANNELED SPECTROPOLARIMETRY}

Channeled spectropolarimetry takes advantage of the wavelength dependence of thick retarders to obtain spectrally-resolved Stokes vector measurements. ${ }^{1}$ By orienting a pair of thick retarders at $45^{\circ}$ from one another, the various elements of the Stokes vector cause interference fringes in the spectrum, where the amplitudes and frequencies of the fringes carry the Stokes parameter information (see Fig. 1).

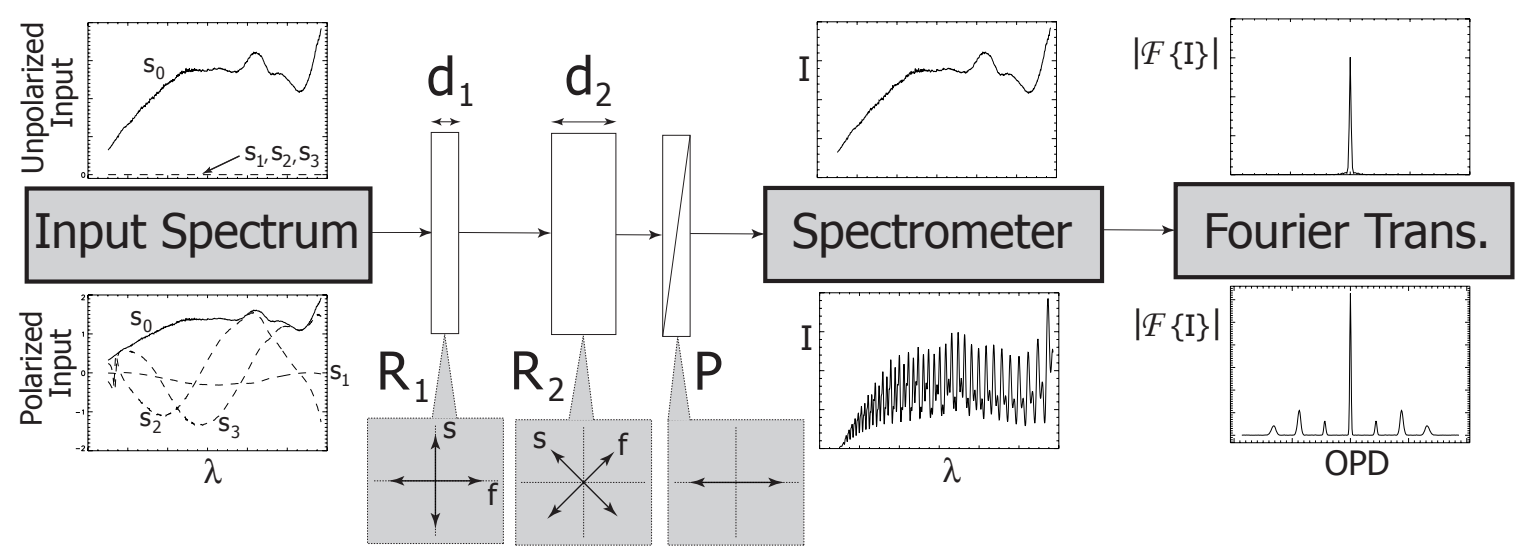

Figure 1: The channeled spectropolarimeter layout, showing two example input spectra and their resulting channeled spectra. In the Fourier domain, we can see the seven channels of data (DC plus three positive and three negative) individually separated along the OPD axis.

If we represent the Mueller matrices of the components with $\mathbf{P}(\phi)$, for a linear polarizer oriented at angle $\phi$ to the horizontal, and $\mathbf{R}(\phi, \delta)$ for a linear retarder oriented at angle $\phi$ with retardance $\delta$, then the Mueller-calculus of the system will take an input Stokes vector $\mathbf{s}_{\mathrm{in}}=\left(\begin{array}{llll}s_{0} & s_{1} & s_{2} & s_{3}\end{array}\right)^{\top}$ and produce an output spectrum $I_{\text {out }}$ of $^{2}$

$$
\left.\begin{array}{rl}
I_{\text {out }}(\lambda)= & \mathbf{d} \cdot \mathbf{P}\left(0^{\circ}\right) \cdot \mathbf{R}\left(45^{\circ}, \delta_{2}\right) \cdot \mathbf{R}\left(0^{\circ}, \delta_{1}\right) \cdot \mathbf{s}_{\text {in }} \\
= & \frac{1}{2}\left[s_{0}+s_{1} \cos \left(\delta_{2}\right)+s_{2} \sin \left(\delta_{1}\right) \sin \left(\delta_{2}\right)-s_{3} \cos \left(\delta_{1}\right) \sin \left(\delta_{2}\right)\right] \\
= & \frac{1}{2}\left[s_{0}+\frac{1}{4} s_{1}\left(e^{i \delta_{2}}+e^{-i \delta_{2}}\right)\right. \\
& \quad+\frac{1}{8}\left[\left(s_{2}-i s_{3}\right) e^{i\left(\delta_{2}+\delta_{1}\right)}+\left(s_{2}+i s_{3}\right) e^{-i\left(\delta_{2}+\delta_{1}\right)}+\left(-s_{2}-i s_{3}\right) e^{i\left(\delta_{2}-\delta_{1}\right)}+\left(-s_{2}+i s_{3}\right) e^{-i\left(\delta_{2}-\delta_{1}\right)}\right],
\end{array}\right\}
$$

Polarization Science and Remote Sensing IX, edited by Julia M. Craven, Joseph A. Shaw, Frans Snik, Proc. of SPIE Vol. 11132, 111320H · @ $2019 \mathrm{SPIE} \cdot \mathrm{CCC}$ code: 0277-786X/19/\$21 · doi: 10.1117/12.2525006 


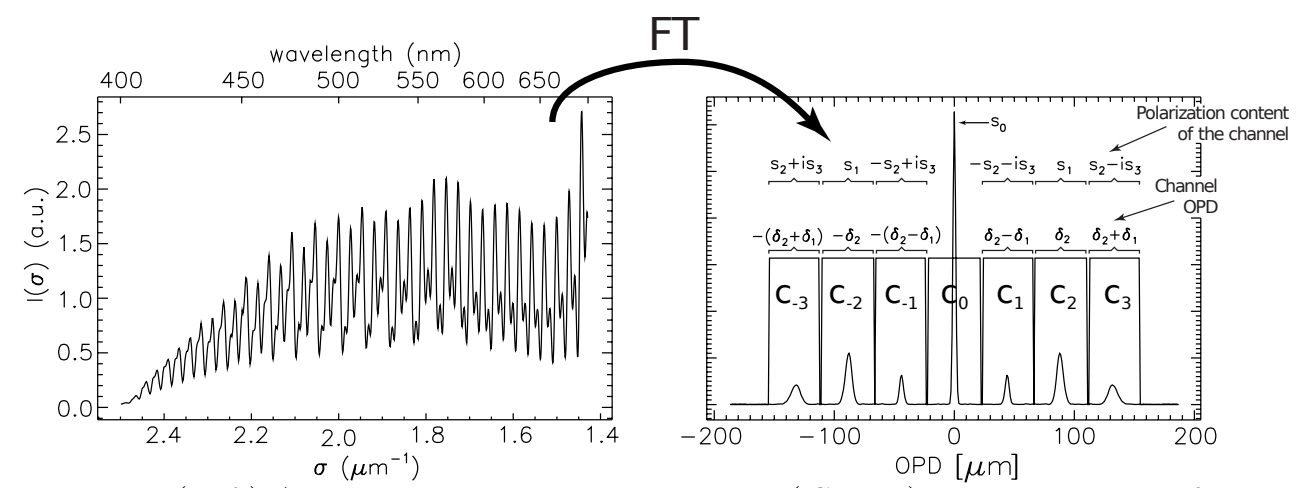

\begin{tabular}{rccl}
\hline$C_{n}$ & $\delta_{1}$ & $\delta_{2}$ & contents \\
\hline 0 & 0 & 0 & $s_{0}$ \\
1 & - & + & $s_{2}-i s_{3}$ \\
2 & 0 & + & $s_{1}$ \\
3 & + & + & $s_{2}-i s_{3}$ \\
-1 & + & - & $-s_{2}+i s_{3}$ \\
-2 & 0 & - & $s_{1}$ \\
-3 & - & - & $s_{2}+i s_{3}$ \\
\hline
\end{tabular}

Figure 2: (Left) An example channeled spectrum. (Center) The magnitude of the channeled spectrum's Fourier transform. Window functions have been superimposed onto the plot to indicate the seven channels. (Right) A table showing the relationship between the OPDs $\delta_{i}$ of the two retarders and each channel's encoded polarization content.

where $\mathbf{d}=\left(\begin{array}{llll}1 & 0 & 0 & 0\end{array}\right)$ is the detection row vector. Note that all of the $s_{i}$ and $\delta_{i}$ in this equation are wavelengthdependent. From this result, we can see that the four Stokes components are each encoded into the intensity spectrum, where the data is modulated into seven heterodyne frequencies, centered at OPD values of: $0, \pm \delta_{2}$, $\pm\left(\delta_{2}+\delta_{1}\right)$, and $\pm\left(\delta_{2}-\delta_{1}\right)$. If we take the Fourier transform of this spectrum with respect to wavenumber $\sigma=1 / \lambda)$, then we obtain the 7-channel distribution shown at the far right of Fig. 1. By filtering the Fourier domain (OPD) spectrum, we can reconstruct the individual Stokes component spectra $s_{i}(\sigma)$.

The seven channels of data are centered at heterodyne frequencies determined by the OPDs of the retarders. In order to locate the positions of the channels, we write the expression for the retardance OPD as a function of wavenumber, given by

$$
\delta(\sigma)=2 \pi t B(\sigma) \sigma
$$

for a plate of thickness $t$, of birefringence $B(\sigma)=n_{\mathrm{e}}(\sigma)-n_{\mathrm{o}}(\sigma)$, at wavenumber $\sigma$. Because the birefringence is also wavelength-dependent, we can rewrite this expression as ${ }^{1}$

$$
\delta(\sigma)=2 \pi t\left(B\left(\sigma_{0}\right)+\left[\frac{\partial B}{\partial \sigma}\right]_{\sigma_{0}} \sigma_{0}\right) \sigma
$$

where $\sigma_{0}$ is the central wavenumber of the spectrum: $\sigma_{0}=\frac{1}{2}\left(\sigma_{\min }+\sigma_{\max }\right)$.

For quartz retarders, a useful rule of thumb is that the OPD is approximately $0.8 \%$ of the thickness of the crystal, when operating in the visible spectral range. Different sources give different values for the birefringence, so it is best to measure the value directly. However, by the $0.8 \%$ rule, we can say that a $6.6 \mathrm{~mm}$ thick retarder produces roughly $53 \mu \mathrm{m}$ of OPD. Most of this OPD is determined by the average birefringence term $B\left(\sigma_{0}\right)$, while the gradient term $\partial B / \partial \sigma$ reduces the sum by about $9 \%$ is due to negative slope with respect to increasing wavenumber.

From the OPD values, we can also determine the resolution of the reconstructed Stokes spectra. For the 7-channel system shown in Fig. 2, the spectral data is split by a factor of 7 between the available channels. Conventional spectrometers are typically designed to oversample the spectrum in order to prevent aliasing and to improve light collection. If we assume that the sampling is 4 times the Nyquist rate, then we can only resolve out to one-fourth of the full OPD domain, so that a 4000-element detector generates a spectrum with no more than 1000 resolution elements. Thus, a 1000-resolution-element spectrum split between 7 channels of data will allow up to $1000 / 7=143$ resolvable spectral elements per reconstructed channel. If we know the spectral range and spectral resolution of our spectrometer, then we can design the retarder OPDs to fill out this domain for optimal measurement bandwidth. 


\section{SNAPSHOT MUELLER MATRIX SPECTROPOLARIMETRY}

Recently, channeled spectropolarimetry has been subsumed under the more general category of "channeled polarimeters", ${ }^{3}$ and has also been extended to active (Mueller matrix) spectropolarimetry. ${ }^{4}$ For snapshot Muellermatrix spectropolarimetry (SMSP), we employ two channeled spectropolarimeters: a high-resolution CHSP on the analysis side, for measuring the spectrum exiting the test sample, and a lower-resolution CHSP operating in reverse for illumination, so that the polarization state incident on the sample is modulated along the wavelength axis. If the Mueller matrix of the sample varies only slowly with wavelength, then the measurements will not overlap in the OPD domain and can be accurately recovered. Figure 3 shows the resulting system layout, with polarizers $P_{1}$ and $P_{2}$, and retarders $R_{1}, R_{2}, R_{3}$, and $R_{4}$.

Taking the same approach as (1), we assume an unpolarized light source of intensity $I_{0}$ so that $\mathbf{s}_{\mathrm{in}}(\sigma)=$ $\left(\begin{array}{llll}I_{0}(\sigma) & 0 & 0 & 0\end{array}\right)^{\top}$. The intensity at the spectrometer is therefore given by

$$
I_{\text {out }}(\sigma)=\mathbf{d} \mathbf{P}_{2}\left(0^{\circ}\right) \mathbf{R}_{4}\left(45^{\circ}, \delta_{4}\right) \mathbf{R}_{3}\left(0^{\circ}, \delta_{3}\right) \mathbf{M} \mathbf{R}_{2}\left(0^{\circ}, \delta_{2}\right) \mathbf{R}_{1}\left(45^{\circ}, \delta_{1}\right) \mathbf{P}_{1}\left(0^{\circ}\right) \mathbf{s}_{\text {in }}(\sigma),
$$

where $\mathbf{M}$ is the sample Mueller matrix. Writing out (2) in terms of the individual Mueller matrix elements gives

$$
\begin{aligned}
I(\sigma)=\frac{I_{0}(\sigma)}{4}( & m_{00}+m_{01} \cos \delta_{1}+m_{10} \cos \delta_{4} \\
& +m_{02} \cos \delta_{1} \sin \delta_{2}+m_{03} \cos \delta_{2} \sin \delta_{1}+m_{11} \cos \delta_{1} \cos \delta_{4}+m_{20} \sin \delta_{3} \sin \delta_{4}-m_{30} \cos \delta_{3} \sin \delta_{4} \\
& +m_{12} \sin \delta_{1} \sin \delta_{2} \cos \delta_{4}+m_{13} \sin \delta_{1} \cos \delta_{2} \cos \delta_{4}+m_{21} \cos \delta_{1} \sin \delta_{3} \sin \delta_{4} \\
& +m_{22} \sin \delta_{1} \sin \delta_{2} \sin \delta_{3} \sin \delta_{4}+m_{23} \sin \delta_{1} \cos \delta_{2} \sin \delta_{3} \sin \delta_{4}-m_{31} \cos \delta_{1} \cos \delta_{3} \sin \delta_{4} \\
& \left.-m_{32} \sin \delta_{1} \sin \delta_{2} \cos \delta_{3} \sin \delta_{4}-m_{33} \sin \delta_{1} \cos \delta_{2} \cos \delta_{3} \sin \delta_{4}\right) .
\end{aligned}
$$

With the proper choice of retarder thicknesses, the various matrix elements are separated into independent channels in the Fourier domain, as for example shown in Fig. 4. Inspired by the dual-rotating-retarder Mueller matrix polarimeter, ${ }^{5}$ this example shows the channels for a $5: 1$ ratio of thicknesses for the pair of analyzing retarders to the generating pair, and a 2:1 ratio of thicknesses for the retarders within each pair. Thus, we designate this as a 1:2:5:10 configuration, for the relative thicknesses of $R_{1}$ through $R_{4}$.

The 1:2:5:10 configuration, however, is only one example of many possible configurations. To develop a more general structure for analyzing the possible designs, we can list the various possibilities as shown in Table 1 . This structure is similar to one developed by Alenin and Tyo for analyzing channeled polarimeters. ${ }^{3}$ Here we leave the values of the $\delta_{i}$ undefined as yet, and look at the various possible combinations, with the constraint that a given channel OPD can be formed by summing, differencing, or ignoring each of the retardances in turn.

From Table 1 we can see that there are 49 possible channels containing data. The advantage of using this table is that we can simply substitute into the four modulation factors $\delta_{i}$ in order to get the OPD value of each of the 49 total channels, for any given configuration of retarder thicknesses. Some other possible choices that are of particular interest include: 1:1:5:5 (suggested by Dubreuil et al. ${ }^{6}$ ), 2:1:4:11 and 3:1:3:2 (both suggested by Alenin and $\mathrm{Tyo}^{3}$ ), and two new configurations 2:1:7:15 and 1:2:7:15. Table 2 lists the various OPD values generated by these configuration choices. At the top of the table are two rows summarizing the characteristics of

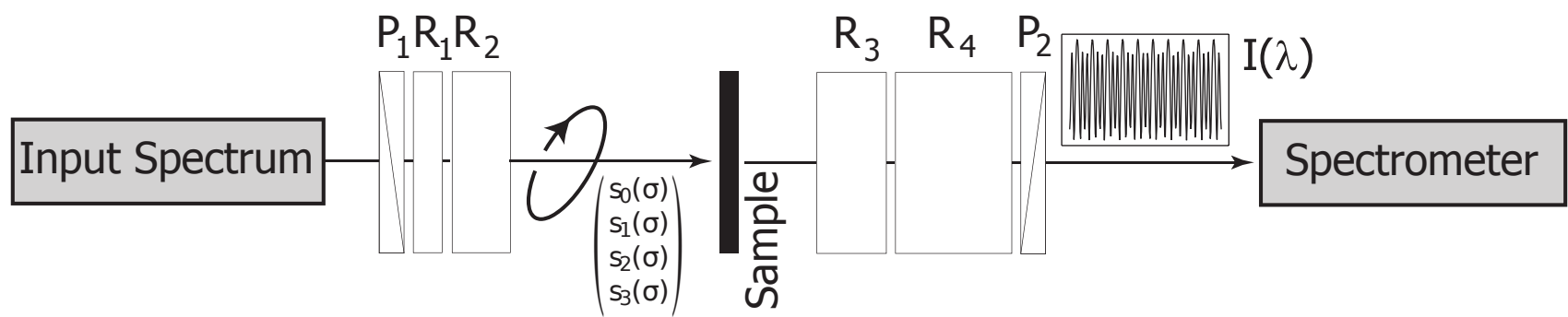

Figure 3: The basic layout of the snapshot Mueller matrix spectropolarimeter. Retarders $R_{1} \& R_{4}$ have their fast axes oriented at $45^{\circ}$, retarders $R_{2} \& R_{3}$ at $0^{\circ}$. Polarizers $P_{1} \& P_{2}$ both have their transmission axes oriented at $0^{\circ}$. 


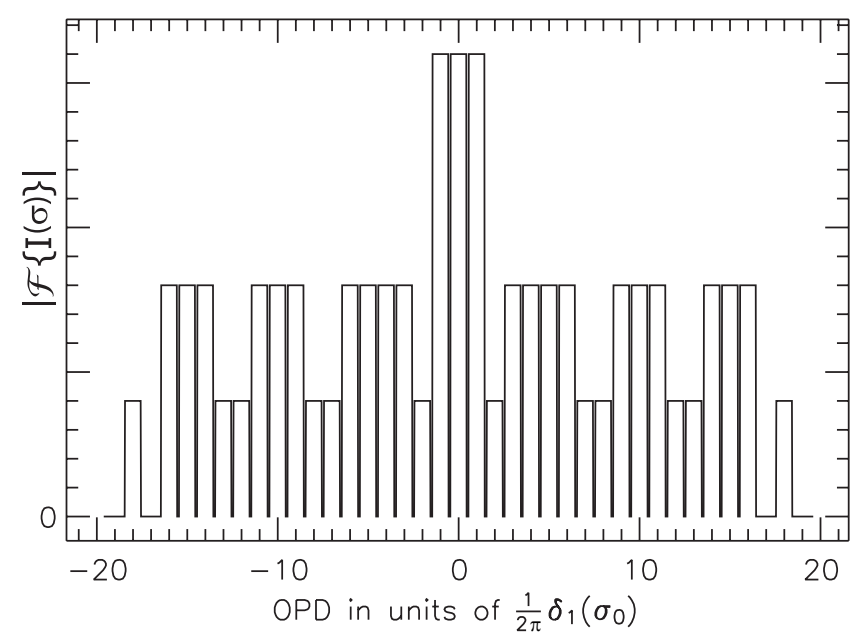

Figure 4: The 37 channels $C_{n}$ in the Fourier domain for the 1:2:5:10 configuration. The OPD axis is in multiples of the OPD of the thinnest retarder. The heights of the channels shown here indicate the channel amplitudes for unit inputs.

each configuration, labelled "A" and "B", giving the number of "multiplex channels", and the maximum OPD respectively.

Looking along row "B", we can see that among these designs, the 3:1:3:2 system provides the smallest overall bandwidth, using a maximum OPD of only 9 times the thinnest retarder. The 1:2:5:10 design requires twice that, at 18 times, and the 2:1:7:15 requires the largest amount, at 25 times the thinnest retarder. Another important consideration, however, is the effect of multiplexing on the signal-to-noise ratio (SNR) of the reconstructed spectra. Alenin and Tyo pointed out that although the 3:1:3:2 design provides the smallest overall bandwidth, it is also noisy. The originally proposed 1:2:5:10 design is likewise noisy, but swapping the first two channels (i.e., a 2:1:5:10 design) halves the noise in the reconstructed data.3 Alenin and Tyo suggest using a 2:1:4:11 design as providing an optimal noise performance.

Here we would like to point out a crucial parameter that has not been discussed before: the multiplex factor given in column "A". This factor describes the number of OPD values in the Fourier domain for which there are multiple pathways through the table to reach the same OPD value. This is a problem for robustness because a small thickness error in one retarder with respect to its design value can cause one pathway to this OPD (one encoding of the Mueller matrix elements) to shift relative to the other, so that extracting one without causing error in the other is a difficult task. Looking at row "A", we see that here too the 3:1:3:2 design is problematic, with 15 multiplexed channels. This forces the system designer to require tight tolerances on the thickness of all four waveplates in order to achieve good Stokes spectra estimates. The 1:2:5:10 and 2:1:4:11 designs are better, having only 7 and 6 multiplexed channels, respectively, but the multiplexing problem remains.

Among the designs we have analyzed so far, a 2:1:7:15 design appears to have the lowest overall OPD without multiplexed channels, and thus may be considered an optimal configuration for tolerancing purposes. Table 2 also lists the 1:2:7:15 design, in which the first two retarders are swapped, but this one has 7 multiplexed channels while the former design has none. Achieving this robustness to manufacturing tolerances, however, comes at a price in bandwidth: the system needs to resolve the channeled spectrum out to an OPD value of 25 times that of the thinnest retarder, while the reconstructed spectra are limited in bandwidth to the OPD of the thinnest retarder. Fortunately, however, we can say that the noise performance is optimal. Since minimizing multiplexing also minimizes the number of channels that need to be combined in order to separate out each individual Mueller matrix element, the 2:1:7:15 design provides the same minimum noise value achieved by Alenin's 2:1:4:11 setup.

Returning to the example spectrum used for the channeled spectropolarimeter, we can make the bandwidth constraints more concrete. If we have a spectrometer with a resolving power of 1000 and employ this for a 2:1:7:15 design SMSP, then the data will be split into 51 separate channels, giving a maximum resolution in 
Table 1: General mapping between retardances and the Mueller matrix element data contained in each channel for a 4-retarder SMSP. The "\#" column gives the "modulation path number".

\begin{tabular}{|c|c|c|c|c|c|c|c|c|c|c|c|}
\hline$\#$ & $\delta_{1}$ & $\delta_{2}$ & $\delta_{3}$ & $\delta_{4}$ & contents / $\left(64 s_{0}\right)$ & $\#$ & $\delta_{1}$ & $\delta_{2}$ & $\delta_{3}$ & $\delta_{4}$ & contents / $\left(64 s_{0}\right)$ \\
\hline 0 & 0 & 0 & 0 & 0 & $16 m_{00}$ & & & & & & \\
\hline 1 & - & 0 & 0 & 0 & $+8 m_{01}$ & 25 & - & 0 & - & - & $-2 m_{21}-2 i m_{31}$ \\
\hline 2 & + & 0 & 0 & 0 & $+8 m_{01}$ & 26 & + & 0 & - & - & $-2 m_{21}-2 i m_{31}$ \\
\hline 3 & 0 & 0 & 0 & - & $+8 m_{10}$ & 27 & - & 0 & + & - & $+2 m_{21}-2 i m_{31}$ \\
\hline 4 & 0 & 0 & 0 & + & $+8 m_{10}$ & 28 & + & 0 & + & - & $+2 m_{21}-2 i m_{31}$ \\
\hline 5 & - & - & 0 & 0 & $-4 m_{02}+4 i m_{03}$ & 29 & - & 0 & - & + & $+2 m_{21}+2 i m_{31}$ \\
\hline 6 & + & - & 0 & 0 & $+4 m_{02}-4 i m_{03}$ & 30 & + & 0 & - & + & $+2 m_{21}+2 i m_{31}$ \\
\hline 7 & - & + & 0 & 0 & $+4 m_{02}+4 i m_{03}$ & 31 & - & 0 & + & + & $-2 m_{21}+2 i m_{31}$ \\
\hline 8 & + & + & 0 & 0 & $-4 m_{02}-4 i m_{03}$ & 32 & + & 0 & + & + & $-2 m_{21}+2 i m_{31}$ \\
\hline 9 & - & 0 & 0 & - & $+4 m_{11}$ & 33 & - & - & - & - & $+m_{22}+m_{33}-i m_{23}+i m_{32}$ \\
\hline 10 & + & 0 & 0 & - & $+4 m_{11}$ & 34 & + & - & - & - & $-m_{22}-m_{33}+i m_{23}-i m_{32}$ \\
\hline 11 & - & 0 & 0 & + & $+4 m_{11}$ & 35 & - & + & - & - & $-m_{22}+m_{33}-i m_{23}-i m_{32}$ \\
\hline 12 & + & 0 & 0 & + & $+4 m_{11}$ & 36 & + & + & - & - & $+m_{22}-m_{33}+i m_{23}+i m_{32}$ \\
\hline 13 & 0 & 0 & - & - & $-4 m_{20}-4 i m_{30}$ & 37 & - & - & + & - & $-m_{22}+m_{33}+i m_{23}+i m_{32}$ \\
\hline 14 & 0 & 0 & + & - & $+4 m_{20}-4 i m_{30}$ & 38 & + & - & + & - & $+m_{22}-m_{33}-i m_{23}-i m_{32}$ \\
\hline 15 & 0 & 0 & - & + & $+4 m_{20}+4 i m_{30}$ & 39 & - & + & + & - & $+m_{22}+m_{33}+i m_{23}-i m_{32}$ \\
\hline 16 & 0 & 0 & + & + & $-4 m_{20}+4 i m_{30}$ & 40 & + & + & + & - & $-m_{22}-m_{33}-i m_{23}+i m_{32}$ \\
\hline 17 & - & - & 0 & - & $-2 m_{12}+2 i m_{13}$ & 41 & - & - & - & + & $-m_{22}-m_{33}+i m_{23}-i m_{32}$ \\
\hline 18 & + & - & 0 & - & $+2 m_{12}-2 i m_{13}$ & 42 & + & - & - & + & $+m_{22}+m_{33}-i m_{23}+i m_{32}$ \\
\hline 19 & - & + & 0 & - & $+2 m_{12}+2 i m_{13}$ & 43 & - & + & - & + & $+m_{22}-m_{33}+i m_{23}+i m_{32}$ \\
\hline 20 & + & + & 0 & - & $-2 m_{12}-2 i m_{13}$ & 44 & + & + & - & + & $-m_{22}+m_{33}-i m_{23}-i m_{32}$ \\
\hline 21 & - & - & 0 & + & $-2 m_{12}+2 i m_{13}$ & 45 & - & - & + & + & $+m_{22}-m_{33}-i m_{23}-i m_{32}$ \\
\hline 22 & + & - & 0 & + & $+2 m_{12}-2 i m_{13}$ & 46 & + & - & + & + & $-m_{22}+m_{33}+i m_{23}+i m_{32}$ \\
\hline 23 & - & + & 0 & + & $+2 m_{12}+2 i m_{13}$ & 47 & - & + & + & + & $-m_{22}-m_{33}-i m_{23}+i m_{32}$ \\
\hline 24 & + & + & 0 & + & $-2 m_{12}-2 i m_{13}$ & 48 & + & + & + & + & $+m_{22}+m_{33}+i m_{23}-i m_{32}$ \\
\hline
\end{tabular}

the Mueller matrix element spectra of 19 elements. For non-aliased measurements, each Mueller matrix element spectrum should be bandlimited to this extent.

\section{CONCLUSION}

In addition to the signal-to-noise ratio in the reconstruction, and the resolution bandlimit, we have shown a third criterion important to the design of a snapshot Mueller matrix spectropolarimeter. This criterion is not an issue for channeled spectropolarimeters, since the limited number of channels there means that each channel is formed from taking only one unique pathway through the retarders. In the SMSP, however, the same OPD can be achieved through multiple pathways, so that small errors in retarder thicknesses will produce multiplexed data of one heterodyne channel overlapped with another, shifted, heterodyne channel. Avoiding this places tight tolerancing requirements on the retarder manufacture.

\section{REFERENCES}

1. K. Oka and T. Kato, "Spectroscopic polarimetry with a channeled spectrum," Opt. Lett. 24, 1475-1477 (1999).

2. N. Hagen, "Snapshot imaging spectropolarimetry," Ph.D. dissertation (University of Arizona, 2007).

3. A. S. Alenin and J. S. Tyo, "Generalized channeled polarimetry," J. Opt. Soc. Am. A 31, 1013-1022 (2014).

4. N. Hagen, K. Oka, and E. L. Dereniak, "Snapshot Mueller matrix spectropolarimeter," Opt. Lett. 32, 2100$2102(2007)$.

5. R. M. A. Azzam, "Photopolarimetric measurement of the Mueller matrix by Fourier analysis of a single detected signal," Opt. Lett. 2, 148-150 (1978).

6. M. Dubreuil, S. Rivet, B. L. Jeune, and J. Cariou, "Snapshot Mueller matrix polarimeter by wavelength polarization coding," Opt. Express 15, 13660-13668 (2007). 
Table 2: The various OPDs obtained by substituting a set of four retarder thicknesses into Table 1, given in units of the thinnest retarder's mean OPD. Row "A" represents the number of multiplex channels, and row "B" represents the maximum OPD, produced by the resulting system.

\begin{tabular}{|c|c|c|c|c|c|c|}
\hline & 2:1:4:11 & $1: 2: 5: 10$ & $1: 1: 5: 5$ & $3: 1: 3: 2$ & $2: 1: 7: 15$ & $1: 2: 7: 15$ \\
\hline A: & 6 & 7 & 12 & 15 & 0 & 7 \\
\hline B: & 18 & 18 & 12 & 9 & 25 & 25 \\
\hline 0 & 0 & 0 & 0 & 0 & 0 & 0 \\
\hline 1 & -2 & -1 & -1 & -3 & -2 & -1 \\
\hline 2 & 2 & 1 & 1 & 3 & 2 & 1 \\
\hline 3 & -3 & -3 & -2 & -4 & -3 & -3 \\
\hline 4 & 1 & -1 & 0 & 2 & 1 & -1 \\
\hline 5 & -1 & 1 & 0 & -2 & -1 & 1 \\
\hline 6 & 3 & 3 & 2 & 4 & 3 & 3 \\
\hline 7 & -11 & -10 & -5 & -2 & -15 & -15 \\
\hline 8 & 11 & 10 & 5 & 2 & 15 & 15 \\
\hline 9 & -13 & -11 & -6 & -5 & -17 & -16 \\
\hline 10 & -9 & -9 & -4 & 1 & -13 & -14 \\
\hline 11 & 9 & 9 & 4 & -1 & 13 & 14 \\
\hline 12 & 13 & 11 & 6 & 5 & 17 & 16 \\
\hline 13 & -14 & -13 & -7 & -6 & -18 & -18 \\
\hline 14 & -10 & -11 & -5 & 0 & -14 & -16 \\
\hline 15 & -12 & -9 & -5 & -4 & -16 & -14 \\
\hline 16 & -8 & -7 & -3 & 2 & -12 & -12 \\
\hline 17 & 8 & 7 & 3 & -2 & 12 & 12 \\
\hline 18 & 12 & 9 & 5 & 4 & 16 & 14 \\
\hline 19 & 10 & 11 & 5 & 0 & 14 & 16 \\
\hline 20 & 14 & 13 & 7 & 6 & 18 & 18 \\
\hline 21 & -15 & -15 & -10 & -5 & -22 & -22 \\
\hline 22 & -7 & -5 & 0 & 1 & -8 & -8 \\
\hline 23 & 7 & 5 & 0 & -1 & 8 & 8 \\
\hline 24 & 15 & 15 & 10 & 5 & 22 & 22 \\
\hline 25 & -17 & -16 & -11 & -8 & -24 & -23 \\
\hline 26 & -13 & -14 & -9 & -2 & -20 & -21 \\
\hline 27 & -9 & -6 & -1 & -2 & -10 & -9 \\
\hline 28 & -5 & -4 & 1 & 4 & -6 & -7 \\
\hline 29 & 5 & 4 & -1 & -4 & 6 & 7 \\
\hline 30 & 9 & 6 & 1 & 2 & 10 & 9 \\
\hline 31 & 13 & 14 & 9 & 2 & 20 & 21 \\
\hline 32 & 17 & 16 & 11 & 8 & 24 & 23 \\
\hline 33 & -18 & -18 & -12 & -9 & -25 & -25 \\
\hline 34 & -14 & -16 & -10 & -3 & -21 & -23 \\
\hline 35 & -16 & -14 & -10 & -7 & -23 & -21 \\
\hline 36 & -12 & -12 & -8 & -1 & -19 & -19 \\
\hline 37 & -10 & -8 & -2 & -3 & -11 & -11 \\
\hline 38 & -6 & -6 & 0 & 3 & -7 & -9 \\
\hline 39 & -8 & -4 & 0 & -1 & -9 & -7 \\
\hline 40 & -4 & -2 & 2 & 5 & -5 & -5 \\
\hline 41 & 4 & 2 & -2 & -5 & 5 & 5 \\
\hline 42 & 8 & 4 & 0 & 1 & 9 & 7 \\
\hline 43 & 6 & 6 & 0 & -3 & 7 & 9 \\
\hline 44 & 10 & 8 & 2 & 3 & 11 & 11 \\
\hline 45 & 12 & 12 & 8 & 1 & 19 & 19 \\
\hline 46 & 16 & 14 & 10 & 7 & 23 & 21 \\
\hline 47 & 14 & 16 & 10 & 3 & 21 & 23 \\
\hline 48 & 18 & 18 & 12 & 9 & 25 & 25 \\
\hline
\end{tabular}

